\title{
Potential role of vitamin D deficiency on Fabry cardiomyopathy
}

\author{
Christiane Drechsler • Benjamin Schmiedeke • Markus Niemann • Daniel Schmiedeke • \\ Johannes Krämer • Irina Turkin • Katja Blouin • Andrea Emmert • Stefan Pilz • \\ Barbara Obermayer-Pietsch • Frank Weidemann • Frank Breunig • Christoph Wanner
}

Received: 15 January 2013 /Revised: 29 July 2013 / Accepted: 26 August 2013 / Published online: 19 October 2013

(C) The Author(s) 2013. This article is published with open access at Springerlink.com

\begin{abstract}
Patients with Fabry disease frequently develop left ventricular (LV) hypertrophy and renal fibrosis. Due to heat intolerance and an inability to sweat, patients tend to avoid exposure to sunlight. We hypothesized that subsequent vitamin D deficiency may contribute to Fabry cardiomyopathy. This study investigated the vitamin D status and its association with LV mass and adverse clinical symptoms in patients with Fabry disease. 25-hydroxyvitamin $\mathrm{D}(25[\mathrm{OH}] \mathrm{D})$ was measured in 111 patients who were genetically proven to have Fabry disease. LV mass and cardiomyopathy were assessed by magnetic resonance imaging and echocardiography. In cross-sectional
\end{abstract}

Communicated by: Verena Peters

C. Drechsler • B. Schmiedeke • M. Niemann • D. Schmiedeke •

J. Krämer • I. Turkin · K. Blouin · A. Emmert · F. Weidemann •

F. Breunig $\cdot \mathrm{C}$. Wanner

Department of Medicine 1, Divisions of Nephrology and Cardiology,

University Hospital Würzburg, Würzburg, Germany

C. Drechsler $\cdot$ M. Niemann $\cdot$ J. Krämer $\cdot$ F. Weidemann $\cdot$ C. Wanner Comprehensive Heart Failure Center, University of Würzburg,

Würzburg, Germany

C. Drechsler

Department of Clinical Epidemiology, University of Würzburg, Würzburg, Germany

S. Pilz $\cdot$ B. Obermayer-Pietsch

Department of Internal Medicine, Division of Endocrinology and

Metabolism, Medical University of Graz, Graz, Austria

S. Pilz

Department of Epidemiology and Biostatistics, EMGO Institute for Health and Care Research, VU University Medical Centre,

Amsterdam, The Netherlands

C. Drechsler $(\square)$

Department of Medicine, Division of Nephrology, University

Hospital, Oberdürrbacherstr. 6, D-97080 Würzburg, Germany

e-mail: drechsler_c@medizin.uni-wuerzburg.de analyses, associations with adverse clinical outcomes were determined by linear and binary logistic regression analyses, respectively, and were adjusted for age, sex, BMI and season. Patients had a mean age of $40 \pm 13$ years ( $42 \%$ males), and a mean 25(OH)D of $23.5 \pm 11.4 \mathrm{ng} / \mathrm{ml}$. Those with overt vitamin D deficiency $(25[\mathrm{OH}] \mathrm{D} \leq 15 \mathrm{ng} / \mathrm{ml})$ had an adjusted six fold higher risk of cardiomyopathy, compared to those with sufficient $25(\mathrm{OH}) \mathrm{D}$ levels $>30 \mathrm{ng} / \mathrm{ml}(p=0.04)$. The mean LV mass was distinctively different with $170 \pm 75 \mathrm{~g}$ in deficient, $154 \pm$ $60 \mathrm{~g}$ in moderately deficient and $128 \pm 58 \mathrm{~g}$ in vitamin $\mathrm{D}$ sufficient patients $(p=0.01)$. With increasing severity of vitamin D deficiency, the median levels of proteinuria increased, as well as the prevalences of depression, edema, cornea verticillata and the need for medical pain therapy. In conclusion, vitamin D deficiency was strongly associated with cardiomyopathy and adverse clinical symptoms in patients with Fabry disease. Whether vitamin D supplementation improves complications of Fabry disease, requires a randomized controlled trial.

\section{Introduction}

Patients with Fabry disease frequently develop cardiac and renal complications, including progressive nephropathy, transient ischemic attacks, early strokes, hypertrophic cardiomyopathy with arrhythmias, which can subsequently lead to death (Breunig and Wanner 2008; Weidemann et al 2009; Wanner et al 2010; Weidemann et al 2010). The morphological hallmarks of Fabry cardiomyopathy consist of left ventricular hypertrophy and replacement fibrosis. At the advanced stage of replacement fibrosis, left ventricular function is significantly reduced in the fibrotic segments. This structural damage and functional loss can lead to significant heart failure and even end-stage disease.

Vitamin D is associated with decreased cardiovascularrelated morbidity and mortality (Wolf et al 2007; Dobnig et al 
2008; Pilz et al 2008a, b; Mehrotra et al 2009; Drechsler et al 2010; Drechsler et al 2011), possibly by modifying cardiac structure or function; however, firm evidence for either remains lacking. Vitamin D status plays a crucial role for the myocardium and vasculature. Experimental studies identified vitamin $\mathrm{D}$ receptors and expression of $1 \alpha$-hydroxylase in vascular smooth muscle, endothelial cells, and possibly cardiac tissue (Somjen et al 2005; Chen et al 2008; Chen et al 2011). These cells and tissues may therefore be able to produce $1,25(\mathrm{OH}) \mathrm{D}$ on a local level. Experimental data showed that vitamin D receptor knockout and $1 \alpha$-hydroxylase knockout mice develop heart failure, despite having normal calcium levels (Bouillon et al 2008; Pilz et al 2010).

Due to symptoms such as heat intolerance and the inability to sweat, Fabry patients tend to avoid sunlight exposure. Often, patients also suffer from malabsorptive gastrointestinal disease. Both conditions may contribute to a deficiency in vitamin D. It is equally important to note that, patients with Fabry disease have been shown to suffer from low bone mineral density, leading to osteopenia and osteoporosis, further linking vitamin D deficiency to the disease (Germain et al 2005; Mersebach et al 2007). Given the link between vitamin D deficiency and abnormalities in cardiac structure and function, we hypothesized that vitamin D deficiency may contribute to the cardiomyopathy observed in Fabry patients. This study investigated the vitamin D status and its association with left ventricular mass and hypertrophy, as well as adverse clinical symptoms in a large cohort of patients with Fabry disease.

\section{Methods}

\section{Study population}

Between 2001 and 2009, 111 patients with genetically confirmed Fabry disease were seen at the Fabry Centre for Interdisciplinary Therapy in Würzburg and were included into this study upon their first visit. Inclusion criteria for the present study were (1) genetically proven Fabry disease and (2) availability of blood samples for measurement of $25(\mathrm{OH})$ vitamin $\mathrm{D}$. For the analysis, we divided the cohort according to their baseline $25(\mathrm{OH})$ vitamin $\mathrm{D}$ levels into groups based on overt deficient $<15 \mathrm{ng} / \mathrm{ml}(<37 \mathrm{nmol} / \mathrm{l})$, moderately deficient $15-30 \mathrm{ng} / \mathrm{ml}$ and sufficient $>30 \mathrm{ng} / \mathrm{ml}(75 \mathrm{nmol} / \mathrm{l})$ serum levels in line with established categorizations used in the literature (Mehrotra et al 2008; Wang et al 2008). Clinical symptoms and renal parameters were assessed. Parameters of cardiac function were measured by echocardiography and magnetic resonance imaging.

All procedures followed were in accordance with the ethical standards of the responsible committee on human experimentation (institutional and national) and with the Helsinki Declaration of 1975, as revised in 2000. The ethics committee approved the research protocol. Written informed consent was obtained from all patients for being included in the study.

Data collection

Information on age, gender, smoking status and history of hypertension was obtained through patient interviews. Systolic and diastolic blood pressure was measured in sitting position after 5 min rest. Body mass index (BMI) was calculated as weight $(\mathrm{kg})$ divided by height $(\mathrm{m})$ squared. Comorbidities, including the presence of coronary artery disease, congestive heart failure, and cardiac dysrhythmia were obtained from patient reports and the patients' clinical records. In addition, we asked the patients about specific symptoms of Fabry disease, including the presence of anhidrosis, acroparesthesia, heat- and physical stress intolerance, pain, nausea and diarrhea. A physical examination was performed by a medical doctor who was specifically trained in the care of patients with Fabry disease. We recorded information about kidney transplantation and dialysis. Urinary protein excretion was determined in $24 \mathrm{~h}$ urine samples.

Levels of 25(OH)D were measured in serum samples taken at baseline; i.e. prior to any enzyme replacement therapy with galactosidase A. Samples were stored without repeated freeze thaw cycles at $-80{ }^{\circ} \mathrm{C}$ until measurement. Serum concentrations of $25(\mathrm{OH}) \mathrm{D}$ were measured by means of a chemiluminescence assay (IDS, iSYS 25(OH)D; Immunodiagnostic systems Ltd, Boldon, UK) on an IDS-iSYS multi-discipline automated analyser. Within-day coefficients of variation were $5.5-12.1 \%$ and inter-day coefficients of variation were $8.9-16.9 \%$, respectively. Routine haematological and biochemical parameters were all determined in a central laboratory at the Würzburg University hospital. The Glomerular filtration rate was measured by the clearance of Technetium-99m labeled DTPA $\left({ }^{99} \mathrm{Tc}\right.$ diethylenetriaminepentaacetic acid).

MR-tomography and standard echocardiographic measurements

A routine cine-MRI was performed in 81 patients as part of the standard assessment (LV heart mass and MR-ejection fraction) on a Siemens Sonata-Avanto (Erlangen, Germany) or a Philips Gyroscan ACS-NT (Best, Netherlands) 1,5 T FullBody-Scanner (Beer et al 2006). Reasons for the missing examinations were claustrophobia, metal implantations or missing consent.

Patients were lying on their left side for the echocardiographic examination. Echocardiography was performed using a GE Vingmed Vivid 7 (Horten, Norway; 3.5 Mhz). LV end-diastolic (LVEDD) and end-systolic dimensions (LVESD), as well as end-diastolic thickness of the posterior wall (LVPWD) and the septum (IVSD), were measured using standard M-mode echocardiographic methods from parasternal LV long axis images. 
The Devereux formula, indexed to body surface area and gender, was used to calculate the LV mass. Normal values for enddiastolic wall thickness (both posterior and septal wall thickness) are $<11 \mathrm{~mm}$ for women and $<12 \mathrm{~mm}$ for men, respectively. Based on these cut-off values, we determined the presence of hypertrophy of the posterior wall, hypertrophy of the septum, and hypertrophy in general within patients. Hypertrophy in general was documented if either posterior or septal hypertrophy or both were present. Normal values for left ventricular mass include 67-162 $\mathrm{g}$ in women and 88-224 $\mathrm{g}$ in men. Fractional shortening was measured (FS=(LVDD-LVSD)/LVDD). Ejection fraction (EF) was calculated using the modified Simpson method. Blood pool pulsed Doppler of the mitral valve inflow was used to extract the ratio of early-to-late diastolic flow velocity (E/A) and the deceleration time as a parameter to evaluate diastolic dysfunction.

\section{Data analysis}

Continuous variables were expressed as mean with standard deviation or median with interquartile range (IQR) as appropriate, and categorical variables were expressed as percentages. Differences between groups were tested using ANOVA for continuous parameters and the chi-square test for categorical parameters, respectively.

The study population was divided into three groups according to their $25(\mathrm{OH}) \mathrm{D}$ status at baseline. Patients were grouped into overt vitamin D deficient $(\leq 15.0 \mathrm{ng} / \mathrm{ml})$, moderately vitamin D deficient ( $>15 \leq 30 \mathrm{ng} / \mathrm{ml})$, and vitamin D sufficient ( $>30 \mathrm{ng} / \mathrm{ml}$ ) (Mehrotra et al 2008; Wang et al 2008). We assessed the association of baseline $25(\mathrm{OH}) \mathrm{D}$ with LV mass, cardiomyopathy and adverse clinical symptoms. The patients with sufficient $25(\mathrm{OH}) \mathrm{D}$ levels were used as the reference group. By logistic regression analyses, odds ratios (ORs) and corresponding $95 \%$ confidence intervals were calculated. The analyses were adjusted for the following confounders: age, sex, BMI and season (summer/ winter). A p-value of $<0.05$ was considered as indicating statistical significance. All p-values are reported as two-sided. Analyses were performed using SPSS version 16.0.

\section{Results}

Patients had a mean age of $40 \pm 13$ years, and 47 (42\%) of the patients were male. The mean $25(\mathrm{OH}) \mathrm{D}$ concentration was $23.5 \pm 11.4 \mathrm{ng} / \mathrm{ml}$. Of all patients, $26 \%$ had overt vitamin D deficiency $(<15 \mathrm{ng} / \mathrm{mL})$ and $47 \%$ had moderate vitamin D deficiency $(>15 \leq 30 \mathrm{ng} / \mathrm{ml})$. Patients had on average a normal BMI (mean $23.5 \pm 4.2 \mathrm{~kg} / \mathrm{m}^{2}$ ) and normal blood pressure (mean 124/81 mmHg). Regarding comorbidities, about one third (32\%) of the patients had elevated blood pressure $(>130 /$ $80 \mathrm{mmHg}$ ), $34 \%$ suffered from heart failure and $9 \%$ had angina pectoris. The mean glomerular filtration rate was normal
$(93 \pm 35 \mathrm{ml} / \mathrm{min})$. On average, patients did not have overt proteinuria ( $89 \mathrm{mg} /$ day). The baseline characteristics of the Fabry patients are presented in Table 1.

Cardiac and renal function by vitamin D status

Of all patients, nine patients ( $8 \%$ ) had a vitamin $\mathrm{D}$ level below $10 \mathrm{ng} / \mathrm{ml}$, and 15 patients (14\%) had a vitamin D level below $12 \mathrm{ng} / \mathrm{ml}$. When using $15 \mathrm{ng} / \mathrm{ml}$ as a cutoff for severe vitamin D deficiency, a total of 29 patients $(26 \%)$ were affected and were used for subsequent analyses. Patients with moderate or overt vitamin D deficiency more often had cardiac hypertrophy than patients with sufficient vitamin D status. General cardiac hypertrophy was present in $72 \%$ of the patients with overt vitamin $\mathrm{D}$ deficiency, and in $78 \%$ of the patients with moderate deficiency, while it was present in $35 \%$ of the vitamin D sufficient patients only (Table 2). The mean LV mass was substantially different with $170 \pm 75 \mathrm{~g}$ in overt deficient, $154 \pm$ $60 \mathrm{~g}$ in moderately deficient and $128 \pm 58 \mathrm{~g}$ in vitamin D sufficient patients $(p=0.01)$. Regarding renal status, the percentage of patients with proteinuria $(>150 \mathrm{mg} / 24 \mathrm{~h}$ ) was $35 \%$ in the group of vitamin D sufficient patients, and slightly higher with $48 \%$ and $46 \%$ in the groups with moderate and overt vitamin $\mathrm{D}$ deficiency, respectively.

We performed logistic regression analyses to evaluate the risks of adverse cardiac and renal symptoms controlling for the following confounders: age, sex, BMI and season (summer/winter). By adjusted analyses, patients with overt vitamin $\mathrm{D}$ deficiency $(25[\mathrm{OH}] \mathrm{D} \leq 15 \mathrm{ng} / \mathrm{ml})$ had a 6 fold

Table 1 Characteristics of Morbus Fabry patients

\begin{tabular}{ll}
\hline & $\begin{array}{l}\text { Study cohort } \\
\mathrm{N}=111\end{array}$ \\
\hline Age years & $40 \pm 13$ \\
Sex \% female & 58 \\
25(OH) vitamin D $\mathrm{ng} / \mathrm{ml}$ & $23.5 \pm 11.4$ \\
BMI $\mathrm{kg} / \mathrm{m}^{2}$ & $23.5 \pm 4.2$ \\
Systolic blood pressure $\mathrm{mmHg}$ & $124 \pm 17$ \\
Diastolic blood pressure $\mathrm{mmHg}$ & $81 \pm 11$ \\
Arterial hypertension \% & 32 \\
Cardiovascular disease & \\
$\quad$ Angina \% & 9.1 \\
$\quad$ NYHA I-II \% & 34 \\
Arrhythmia \% & 14.7 \\
Renal status & \\
$\quad$ Serum creatinine $\mathrm{mg} / \mathrm{dl}$ & $1.0 \pm 0.6$ \\
GFR $\mathrm{ml} / \mathrm{min}$ & $93 \pm 35$ \\
Proteinuria $\mathrm{mg} / \mathrm{day}$ & $89(23-597)$
\end{tabular}

Values are presented as means and standard deviation or median and interquartile range

$B M I$ Body mass index; GFR Glomerular filtration rate 
Table 2 Cardiac, renal status and clinical symptoms by vitamin D category

\begin{tabular}{llll}
\hline & \multicolumn{2}{l}{$25(\mathrm{OH})$ vitamin $\mathrm{D} n g / \mathrm{ml}$} & \\
\cline { 2 - 4 } & $\leq 15$ & $>15 \leq 30$ & $>30$ \\
& $\mathrm{~N}=29$ & $\mathrm{~N}=52$ & $\mathrm{~N}=30$ \\
\hline Age years & $37 \pm 10$ & $43 \pm 14$ & $38 \pm 12$ \\
Male sex \% & 52 & 44 & 30 \\
BMI kg/m ${ }^{2}$ & $24.9 \pm 4.9$ & $23.0 \pm 3.8$ & $22.9 \pm 3.7$ \\
Systolic RR $m m H g$ & $124 \pm 17$ & $129 \pm 18$ & $117 \pm 14$ \\
Diastolic RR $m$ mHg & $85 \pm 11$ & $80 \pm 11$ & $81 \pm 11$ \\
LV mass $g$ & $170 \pm 75$ & $154 \pm 60$ & $128 \pm 58$ \\
Hypertrophy septum \% & 64 & 69 & 30 \\
Hypertrophy posterior \% & 54 & 69 & 30 \\
Hypertrophy general \% & 72 & 78 & 35 \\
Ejection fraction \% & 62 & 65 & 60 \\
Proteinuria \% & 46 & 48 & 35 \\
Proteinuria $m g /$ day & $118(30-715)$ & $102(34-602)$ & $66(0-416)$ \\
Clinical symptoms & & & \\
Chronic pain \% & 52 & 48 & 31 \\
Pain therapy \% & 37 & 12 & 11 \\
Depression \% & 17 & 6 & 8 \\
Edema \% & 35 & 22 & 11 \\
Heat intolerance \% & 69 & 57 & 46 \\
Cornea verticillata \% & 50 & 53 & 27 \\
Diarrhea \% & 39 & 30 & 29 \\
\hline & & & \\
\hline
\end{tabular}

Values are presented as means and standard deviation or median and interquartile range or percentages, as appropriate

$L V$ Left ventricular

higher risk of cardiac hypertrophy, compared to those with sufficient 25(OH)D levels $>30 \mathrm{ng} / \mathrm{ml}$ (HR 6.4, $95 \%$ CI 1.1$38.8, p=0.04)$. Similarly, the adjusted risk of septal hypertrophy was six fold increased (HR 6.1, $95 \%$ CI 1.1-36.5, $p=$ 0.04). The odds ratio for heart failure was $2.4(0.6-9.3)$ in overt vitamin D deficiency and $2.9(0.9-9.2)$ in moderate vitamin $\mathrm{D}$ deficiency. We did not find any association between vitamin D deficiency, hypertension or arrhythmia. The results of all further logistic regression analyses are presented in Fig. 1.

\section{Clinical symptoms of vitamin D status}

Furthermore, adverse clinical symptoms more often occurred in patients with vitamin D deficiency, as compared to those with vitamin D sufficiency. Patients with vitamin D deficiency experienced chronic pain, depression, and were often in need of medical pain therapy. Similarly, the prevalences of edema, diarrhea, heat intolerance and cornea verticillata were higher for patients with vitamin D deficiency (Table 2).

Logistic regression analyses were performed to evaluate the risks of adverse clinical symptoms associated with vitamin
D status, adjusting for confounders including age, sex, BMI and season. The results of the logistic regression analyses for the occurrence of clinical symptoms are presented in Fig. 1.

\section{Discussion}

The present study investigated the vitamin D status in patients with Fabry disease. We evaluated the associations of vitamin D status with cardiac and renal complications, as well as with clinical symptoms. We found that vitamin D deficiency was prevalent (73\%) among Fabry patients. Vitamin D deficiency was associated with hypertrophic cardiomyopathy and an increased left ventricular mass. With increasing severity of vitamin D deficiency, the median levels of proteinuria increased, as well as the prevalences of depression, edema, cornea verticillata and the need for medical pain therapy.

In general, vitamin $\mathrm{D}$ from either ultraviolet-B induced synthesis in the skin or from nutritional intake is hydroxylated to 25hydroxyvitamin $\mathrm{D}(25[\mathrm{OH}] \mathrm{D})$ in the liver, circulating up to 1000 fold higher concentrations than the most potent vitamin D metabolite 1,25-dihydroxyvitamin D (1,25[OH]2D) (calcitriol) (Dusso et al 2005). The renal production of $1,25(\mathrm{OH}) 2 \mathrm{D}$ is dependent on substrate availability when circulating $25(\mathrm{OH}) \mathrm{D}$ is low. In patients with chronic diseases, limited sunlight exposure and reduced capacity of the skin to synthesize vitamin D, as well as a loss of vitamin $\mathrm{D}$ binding protein in the urine, are mainly responsible for the high prevalence of depressed 25(OH)D levels (Jacob et al 1984; Wolf et al 2007; Wang et al 2008; Mehrotra et al 2009). Fabry patients typically suffer from so-called Fabry crises due to heat intolerance, especially during the summer. Patients systematically avoid outdoor activities and, thus, are potentially at risk of developing insufficient vitamin $\mathrm{D}$ levels.

The treatment of Fabry disease today focuses on relieving symptoms, supporting organs and arresting disease progression. Enzyme replacement therapy with alpha or beta galactosidase A is administered to patients with Fabry disease, but some patients do not respond well to the treatment. So-called adjunctive therapies and treatment strategies are currently being discussed to support patients with Fabry disease and improve morbidity, mortality and quality of life. In this context, a sufficient blockade of the renin-angiotensin-aldosteron system is widely advocated, but adequate vitamin D storages or supplementation may also be necessary. However, we do not yet advise vitamin D therapy on the basis of our data, although nutritional vitamin D supplementation is widely recommended. The present findings are observational and cannot prove causality.

Interestingly, anti-hypertrophic and anti-proliferative actions of vitamin D metabolites have recently been reported (Pilz et al 2010). In hypertrophic hearts, an increased expression of the VDR has been found in cardiac myocytes and fibroblasts (Chen et al 2008). Experimental studies have found that cardiac myocytes and fibroblasts express the enzymes $1 \alpha$ - 


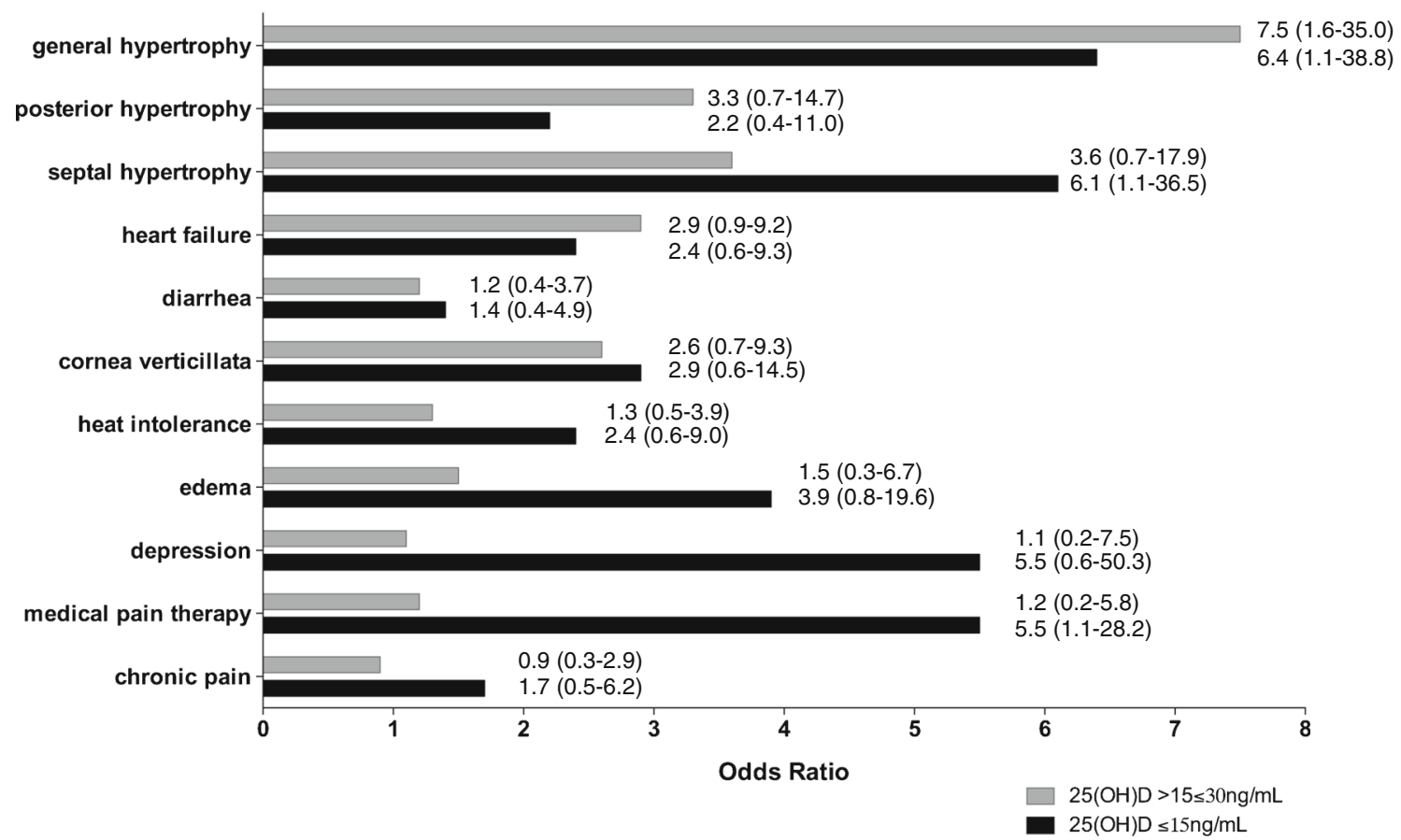

Fig. 1 Odds ratios for the relation between vitamin D status and clinical outcomes, adjusted for age, sex, BMI and season. The odds ratios are presented with $95 \%$ confidence intervals for the patients with overt
25(OH)D deficiency (black) and moderate 25(OH)D deficiency (grey), as compared to the patients with $25(\mathrm{OH}) \mathrm{D}$ sufficiency (reference group)

With increasing severity of vitamin D deficiency, the median levels of proteinuria in our patients increased. Knockout of the vitamin D receptor in diabetic mice was associated with severe albuminuria and glomerulosclerosis from increased thickening of the glomerular basement membrane and podocyte effacement (Zhang et al 2008a, b). Moreover, combined treatment with paricalcitol and an angiotensin receptor blocker in models of diabetic nephropathy prevented the development of albuminuria, maintaining the structure of the glomerular filtration barrier, and reducing glomerulosclerosis, in association with a reduced renal expression of renin (Zhang et al 2008a, b). A recent randomized controlled trial demonstrated a reduction of residual albuminuria in patients with diabetic nephropathy (de Zeeuw et al 2010), whereupon $2 \mu \mathrm{g} /$ day paricalcitol were added to a sufficient inhibition of the renin-angiotensin-aldosteron system.

\section{Conclusion}

In conclusion, our study shows that vitamin D deficiency is associated with the development of complications in patients with Fabry disease, to include progressive Fabry nephropathy, hypertrophic cardiomyopathy and adverse clinical symptoms. It remains to be proven whether vitamin $\mathrm{D}$ deficiency is a risk factor for these complications or merely a marker of disease severity. This is important for the development of novel strategies as additional treatment interventions for patients with Fabry disease. Adjunctive therapeutic strategies can help to support 
patients with Fabry disease and improve morbidity, mortality and quality of life. In this context, novel studies are needed to validate the potential use of vitamin $\mathrm{D}$ status as a marker for disease progression. Importantly, further research is required to establish the role of vitamin D status as potential intervention target. A randomized multicenter controlled trial is required to clarify if vitamin D supplementation improves the complications of Fabry disease.

Acknowledgments We express our gratitude to all patients who participated in this study. We also thank Andrew Lang for helpful discussions and critical revision of the manuscript. The study was supported by the German Ministry of Education and Research (project 01GL0304: Comprehensive Heart Failure Center) and by a fellowship grant from the Medical Faculty of the University of Würzburg (C. Drechsler).

This work was furthermore supported by BioPersMed (COMET Kproject 825329), funded by the Austrian Federal Ministry of Transport, Innovation and Technology (BMVIT), Economics and Labour as well as Economy, Family and Youth (BMWA/BMWFJ) and the Styrian Business Promotion Agency (SFG)

Competing interest Christiane Drechsler, Benjamin Schmiedeke, Markus Niemann, Daniel Schmiedeke, Johannes Krämer, Irina Davydenko, Katja Blouin, Andrea Emmert, Stefan Pilz, Barbara Obermayer-Pietsch, and Frank Breunig declare that they have no conflict of interest.

Frank Weidemann and Christoph Wanner are members of the Fabry Registry European Board of Advisors and have received travel assistance and speaker honoraria from Genzyme-Sanofi and Shire Corporations. Research grants were given to the institution.

Open Access This article is distributed under the terms of the Creative Commons Attribution License which permits any use, distribution, and reproduction in any medium, provided the original author(s) and the source are credited.

\section{References}

Akeno N, Saikatsu S, Kawane T, Horiuchi N (1997) Mouse vitamin D24-hydroxylase: molecular cloning, tissue distribution, and transcriptional regulation by 1 alpha,25-dihydroxyvitamin D3. Endocrinology 138:2233-2240

Bae S, Yalamarti B, Ke Q et al (2011) Preventing progression of cardiac hypertrophy and development of heart failure by paricalcitol therapy in rats. Cardiovasc Res 91:632-639

Beer M, Weidemann F, Breunig F et al (2006) Impact of enzyme replacement therapy on cardiac morphology and function and late enhancement in Fabry's cardiomyopathy. Am J Cardiol 97:1515-1518

Bodyak N, Ayus JC, Achinger S et al (2007) Activated vitamin D attenuates left ventricular abnormalities induced by dietary sodium in Dahl saltsensitive animals. Proc Natl Acad Sci U S A 104:16810-16815

Bouillon R, Carmeliet G, Verlinden L et al (2008) Vitamin D and human health: lessons from vitamin D receptor null mice. Endocr Rev 29:726776

Breunig F, Wanner C (2008) Update on Fabry disease: kidney involvement, renal progression and enzyme replacement therapy. J Nephrol $21: 32-37$

Brown J, Nunez S, Russell M, Spurney C (2009) Hypocalcemic rickets and dilated cardiomyopathy: case reports and review of literature. Pediatr Cardiol 30:818-823
Chen S, Law CS, Grigsby CL et al (2008) Expression of the vitamin d receptor is increased in the hypertrophic heart. Hypertension 52:11061112

Chen S, Law CS, Grigsby CL et al (2011) Cardiomyocyte-specific deletion of the vitamin D receptor gene results in cardiac hypertrophy. Circulation 124:1838-1847

de Zeeuw D, Agarwal R, Amdahl M et al (2010) Selective vitamin D receptor activation with paricalcitol for reduction of albuminuria in patients with type 2 diabetes (VITAL study): a randomised controlled trial. Lancet 376:1543-1551

Dobnig H, Pilz S, Scharnagl H et al (2008) Independent association of low serum 25-hydroxyvitamin $\mathrm{d}$ and 1,25-dihydroxyvitamin $\mathrm{d}$ levels with all-cause and cardiovascular mortality. Arch Intern Med 168:1340-1349

Drechsler C, Pilz S, Obermayer-Pietsch B et al (2010) Vitamin D deficiency is associated with sudden cardiac death, combined cardiovascular events, and mortality in haemodialysis patients. Eur Heart J 31:2253-2261

Drechsler C, Verduijn M, Pilz S et al (2011) Vitamin D status and clinical outcomes in incident dialysis patients: results from the NECOSAD study. Nephrol Dial Transplant 26:1024-1032

Dusso AS, Brown AJ, Slatopolsky E (2005) Vitamin D. Am J Physiol Renal Physiol 289:F8-28

Germain DP, Benistan K, Bououyrie P, Mutschler C (2005) Osteopenia and osteoporosis: previously unrecognized manifestations of Fabry disease. Clin Genet 68:93-95

Jacob AI, Sallman A, Santiz Z, Hollis BW (1984) Defective photoproduction of cholecalciferol in normal and uremic humans. J Nutr 114:1313-1319

Mehrotra R, Kermah D, Budoff M et al (2008) Hypovitaminosis D in chronic kidney disease. Clin J Am Soc Nephrol 3:1144-1151

Mehrotra R, Kermah DA, Salusky IB et al (2009) Chronic kidney disease, hypovitaminosis D, and mortality in the United States. Kidney Int 76: 977-983

Mersebach H, Johansson JO, Rasmussen AK et al (2007) Osteopenia: a common aspect of Fabry disease. Predictors of bone mineral density. Genet Med 9:812-818

Pilz S, Dobnig H, Fischer JE et al (2008a) Low vitamin d levels predict stroke in patients referred to coronary angiography. Stroke 39:2611-2613

Pilz S, Marz W, Wellnitz B et al (2008b) Association of vitamin D deficiency with heart failure and sudden cardiac death in a large cross-sectional study of patients referred for coronary angiography. $\mathrm{J}$ Clin Endocrinol Metab 93:3927-3935

Pilz S, Tomaschitz A, Drechsler C, Dekker JM, Marz W (2010) Vitamin D deficiency and myocardial diseases. Mol Nutr Food Res 54:1103-1113

Przybylski R, McCune S, Hollis B, Simpson RU (2010) Vitamin D deficiency in the spontaneously hypertensive heart failure [SHHF] prone rat. Nutr Metab Cardiovasc Dis 20:641-646

Somjen D, Weisman Y, Kohen F et al (2005) 25-hydroxyvitamin D31alpha-hydroxylase is expressed in human vascular smooth muscle cells and is upregulated by parathyroid hormone and estrogenic compounds. Circulation 111:1666-1671

Tishkoff DX, Nibbelink KA, Holmberg KH, Dandu L, Simpson RU (2008) Functional vitamin D receptor (VDR) in the t-tubules of cardiac myocytes: VDR knockout cardiomyocyte contractility. Endocrinology 149:558-564

Wang TJ, Pencina MJ, Booth SL et al (2008) Vitamin D deficiency and risk of cardiovascular disease. Circulation 117:503-511

Wanner C, Oliveira JP, Ortiz A et al (2010) Prognostic indicators of renal disease progression in adults with Fabry disease: natural history data from the Fabry Registry. Clin J Am Soc Nephrol 5:2220-2228

Weidemann F, Niemann M, Breunig F et al (2009) Long-term effects of enzyme replacement therapy on fabry cardiomyopathy: evidence for a better outcome with early treatment. Circulation 119:524529

Weidemann F, Linhart A, Monserrat L, Strotmann J (2010) Cardiac challenges in patients with Fabry disease. Int J Cardiol 141:3-10 
Wolf M, Shah A, Gutierrez O et al (2007) Vitamin D levels and early mortality among incident hemodialysis patients. Kidney Int 72:1004 1013

Zhang Z, Sun L, Wang Y et al (2008a) Renoprotective role of the vitamin D receptor in diabetic nephropathy. Kidney Int 73:163-171
Zhang Z, Zhang Y, Ning G, Deb DK, Kong J, Li YC (2008b) Combination therapy with AT1 blocker and vitamin D analog markedly ameliorates diabetic nephropathy: blockade of compensatory renin increase. Proc Natl Acad Sci U S A 105: 15896-15901 